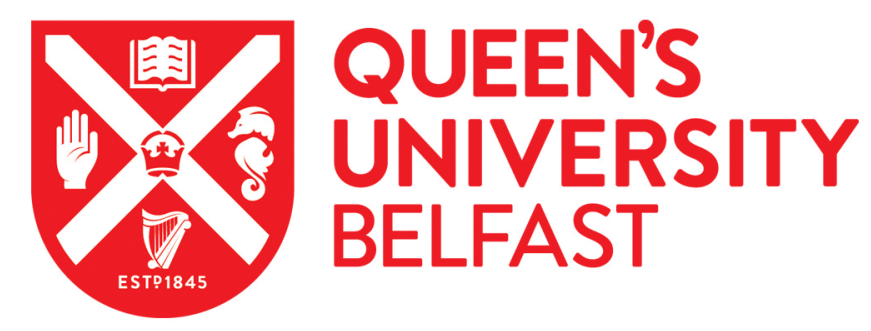

\title{
Role of combination vibrations in resonant positron annihilation
}

Young, J. A., Gribakin, G. F., Lee, C. M. R., \& Surko, C. M. (2008). Role of combination vibrations in resonant positron annihilation. Physical Review A, 77(6), [060702]. https://doi.org/10.1103/PhysRevA.77.060702

Published in:

Physical Review A

Queen's University Belfast - Research Portal:

Link to publication record in Queen's University Belfast Research Portal

\section{General rights}

Copyright for the publications made accessible via the Queen's University Belfast Research Portal is retained by the author(s) and / or other copyright owners and it is a condition of accessing these publications that users recognise and abide by the legal requirements associated with these rights.

Take down policy

The Research Portal is Queen's institutional repository that provides access to Queen's research output. Every effort has been made to ensure that content in the Research Portal does not infringe any person's rights, or applicable UK laws. If you discover content in the Research Portal that you believe breaches copyright or violates any law, please contact openaccess@qub.ac.uk. 


\title{
Role of combination vibrations in resonant positron annihilation
}

\author{
J. A. Young, ${ }^{1, *}$ G. F. Gribakin, ${ }^{2}$ C. M. R. Lee, ${ }^{2}$ and C. M. Surko ${ }^{1}$ \\ ${ }^{1}$ Department of Physics, University of California, San Diego, 9500 Gilman Drive, La Jolla, California 92093-0319, USA \\ ${ }^{2}$ Department of Applied Mathematics and Theoretical Physics, Queen's University, Belfast BT7 INN, Northern Ireland, United Kingdom
}

(Received 15 November 2007; published 27 June 2008)

\begin{abstract}
Positrons can attach to molecules via vibrational Feshbach resonances, leading to very large annihilation rates. The predictions of a recent theory for this process are validated for deuterated methyl halides where all modes are dipole coupled to the incident positron. Data and analysis are presented for methanol and ethylene, demonstrating the importance of combination and overtone resonances and the ability of the theory to account for these features. The mechanism for these resonances and criteria for their occurrence as well as outstanding questions are discussed.
\end{abstract}

DOI: 10.1103/PhysRevA.77.060702

PACS number(s): 34.80.-i, 34.50.-s, 71.60.+z, 78.70.Bj

Positron annihilation by atoms and molecules is important in many situations, including gamma-ray astronomy, materials analysis, and medicine [e.g., positron emission tomography $(\mathrm{PET})]$. In spite of this, we have only begun to understand some of the processes involved in elementary positronmolecule interactions. It is now established that positrons can attach to molecules via vibrational Feshbach resonances (VFRs), resulting in greatly enhanced annihilation rates [1-5]. In this process, the positron makes a transition from a free state to a bound state on the molecule while depositing the excess energy in molecular vibrational modes. This increases greatly the probability of annihilation. Such VFRs also play an important role in low-energy electron attachment to molecules and clusters [6]. Positron energy resolved studies of VFR-mediated processes provide information about the interactions between the positronic, electronic, and nuclear (vibrational) degrees of freedom. Using the unique signature of resonant annihilation, we show that VFRs involving combination vibrations and overtones, i.e., multimode effects, are relatively strong in positron interaction with small molecules.

The annihilation spectra for small molecules with VFR involving infrared-active modes are described by a recent theory [7]. It contains one free parameter, namely, the positron binding energy, which can be determined from experiment [3-5]. So far the theory has been verified for methyl halides [5]. In this paper we use the binding energies found for methyl halides to perform a more stringent test of the theory, by applying it to deuterated methyl halides. The theory is then applied to molecules whose annihilation spectra cannot be explained by simple single-mode resonances. Data and analysis are presented for methanol, demonstrating that VFRs due to combination vibrations and overtones (i.e., multiquantum vibrational excitations) contribute significantly to the annihilation spectrum. By accounting for these effects, we achieve quantitative agreement between the theory and experiment. The more complicated case of ethylene is also analyzed, and the importance of these multimode effects in other molecules is discussed. Finally these results

\footnotetext{
*Present address: Jet Propulsion Laboratory, 4800 Oak Grove Drive, Pasadena, CA 91109, USA.
}

are related to the analogous process of VFR-mediated electron attachment [6].

The procedures used to measure annihilation rates are discussed in detail elsewhere [3-5]. Pulses of positrons from a buffer-gas trap are magnetically guided through a gas cell. Incident positron energies are adjusted by varying the electrical potential of this cell. The distribution of longitudinal positron energies (i.e., parallel to the magnetic field) is approximately $25 \mathrm{meV}$ [full width at half maximum (FWHM)] while the distribution perpendicular to the field is a Maxwellian with a temperature of $\sim 25 \mathrm{meV}[7,8]$. Positrons pass through the gas cell four or five times as annihilation events are recorded, with total scattering kept below 15\%. Annihilation rates are measured with an overall uncertainty of $20 \%$.

Positron annihilation rates, $\lambda$, in gases are typically expressed in terms of dimensionless $Z_{\text {eff }}$, by normalizing to the annihilation rate for an uncorrelated electron gas of the same density, $n$. Thus, $Z_{\text {eff }}=\lambda /\left(\pi r_{0}^{2} c n\right)$, where $c$ is the speed of light and $r_{0}$ is the classical electron radius. It might be expected that $Z_{\text {eff }} \sim Z$, the total number of molecular electrons. However, for many molecules, $Z_{\text {eff }} \gg Z$ due to resonant annihilation [1-5,9-11].

The resonant contribution to $Z_{\text {eff }}$ at the positron beam energy $\varepsilon$ can be expressed as a sum over VFRs [7],

$$
Z_{\mathrm{eff}}^{(\mathrm{res})}(\varepsilon)=\frac{2 \pi}{r_{0}^{2} c} \sum_{\nu} \frac{b_{\nu} \Gamma_{\nu}^{a} \Gamma_{\nu}^{e}}{k_{\nu} \Gamma_{\nu}} \Delta\left(\varepsilon-\varepsilon_{\nu}\right)
$$

where $\Gamma_{\nu}^{e}, \Gamma_{\nu}^{a}$, and $\Gamma_{\nu}$ are the elastic (i.e., capture), annihilation and total widths of the $\nu$ th VFR, $b_{\nu}$ is its multiplicity, and $\Delta\left(\varepsilon-\varepsilon_{\nu}\right)$, which describes the shape of the resonances, is the positron-beam energy distribution [12]. The positron en$\operatorname{ergy} \varepsilon_{\nu}$ and momentum $k_{\nu}=\sqrt{2 \varepsilon_{\nu}}$ (in atomic units) at the $\nu$ th VFR are determined by the vibrational excitation energy $\omega_{\nu}$ and positron binding energy $\varepsilon_{b}$, namely, $\varepsilon_{\nu}=\omega_{\nu}-\varepsilon_{b}$.

For weakly bound, $s$-wave (i.e., fully symmetric) states, the annihilaton width scales as $\Gamma_{\nu}^{a}=\pi r_{0}^{2} c F \sqrt{2 \varepsilon_{b}} /(2 \pi)$ with $F \approx 0.66$ [2]. As a result, the contribution of each resonance to $Z_{\text {eff }}$ is proportional to a simple scale factor $g_{\nu}=\sqrt{\varepsilon_{b} / \varepsilon_{\nu}}$. Beyond this factor, $Z_{\text {eff }}$ involves the dynamics between the positron, electrons, and nuclei, which determine the ratios $\Gamma_{\nu}^{e} / \Gamma_{\nu}$. 


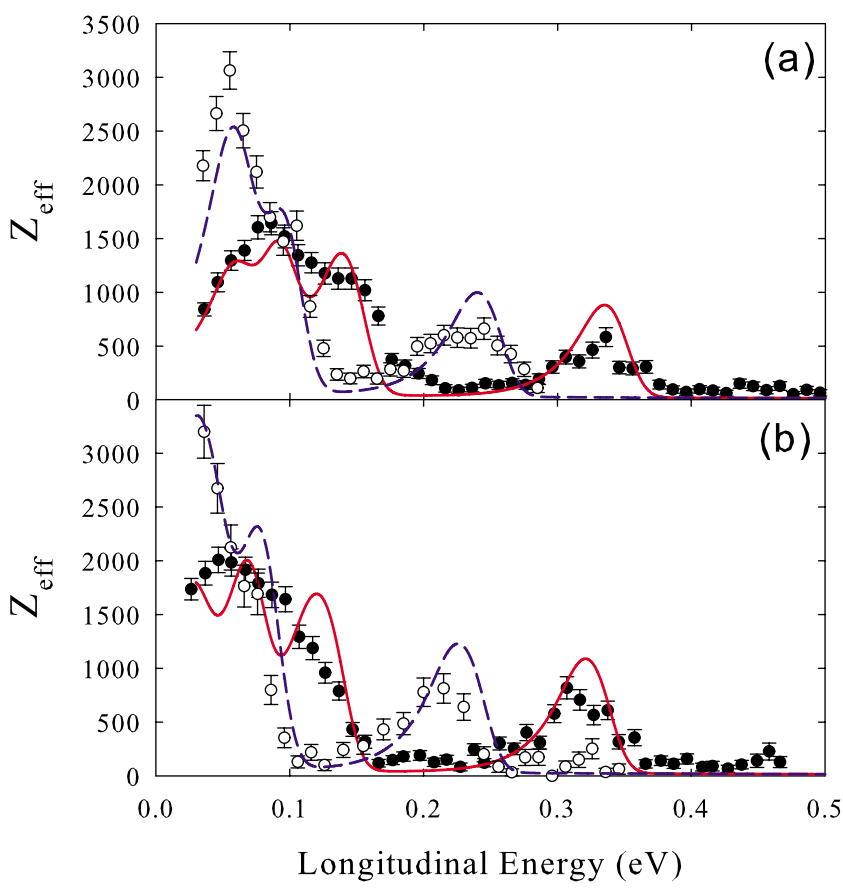

FIG. 1. (Color online) Comparison between experimental (symbols) and theoretical (curves) $Z_{\text {eff }}$ for methyl halides (see $[5,7]$ for details) and their deuterated analogs: (a) $\mathrm{CH}_{3} \mathrm{Cl}$ (solid circles and solid line) and $\mathrm{CD}_{3} \mathrm{Cl}$ (open circles and dashed line), and (b) $\mathrm{CH}_{3} \mathrm{Br}$ (solid circles and solid lines) and $\mathrm{CD}_{3} \mathrm{Br}$ (open circles and dashed lines).

The factor $g_{\nu}$ describes the dependence of the resonant contributions to $Z_{\mathrm{eff}}$ on $\varepsilon_{\nu}$ and $\varepsilon_{b}$ for a broad range of molecules [8]. For infrared-active modes, the elastic widths, $\Gamma_{\nu}^{e}$, can be estimated in the Born-dipole approximation [7], using measured values of infrared (IR) absorption strengths [13]. In methyl halides $\left(\mathrm{CH}_{3} X\right.$, where $X$ is a halogen atom), all vibrational modes are IR active, and $\Gamma_{\nu}^{a} \ll \Gamma_{\nu}^{e}<1 \mathrm{meV}$ [7]. Assuming that positrons are only captured into VFRs of the fundamentals, and using $\Gamma_{\nu}=\Gamma_{\nu}^{e}+\Gamma_{\nu}^{a} \simeq \Gamma_{\nu}^{e}$, one obtains

$$
Z_{\mathrm{eff}}^{(\mathrm{res})}(\varepsilon) \simeq \pi F \sum_{\nu} b_{\nu} g_{\nu} \Delta\left(\varepsilon-\varepsilon_{\nu}\right)
$$

Here each VFR is independent of $\Gamma_{\nu}^{e}$, and $Z_{\mathrm{eff}}^{(\mathrm{res})}$ depends only on the mode energies and the scaling factor $g_{\nu}$. Thus, the remaining free parameter is the binding energy, $\varepsilon_{b}$, which affects the energies of resonances through $\varepsilon_{\nu}$ and their magnitudes through $g_{\nu}$. As shown in Fig. 1, the theory (solid curves) provides good fits to the $\mathrm{Z}_{\text {eff }}$ data for $\mathrm{CH}_{3} \mathrm{Cl}$ and $\mathrm{CH}_{3} \mathrm{Br}$ (full circles), when the binding energies of 25 and $40 \mathrm{meV}$, respectively, are used [5,7].

Experiments show that $\varepsilon_{b}$ does not change with deuteration [3]. At the same time, some mode frequencies in the deuterated species decrease by $\sim 25 \%$ or more. Using the binding energies determined from the experiment for $\mathrm{CH}_{3} \mathrm{Cl}$ and $\mathrm{CH}_{3} \mathrm{Br}$, theoretical predictions were made for $\mathrm{CD}_{3} \mathrm{Cl}$ and $\mathrm{CD}_{3} \mathrm{Br}$ [14] (Fig. 1, dashed curves). Lowering of the mode energies leads to growth of the resonance peak heights, as described by the factor $g_{v}$. The $Z_{\text {eff }}$ for $\mathrm{CD}_{3} \mathrm{Cl}$ and $\mathrm{CD}_{3} \mathrm{Br}$

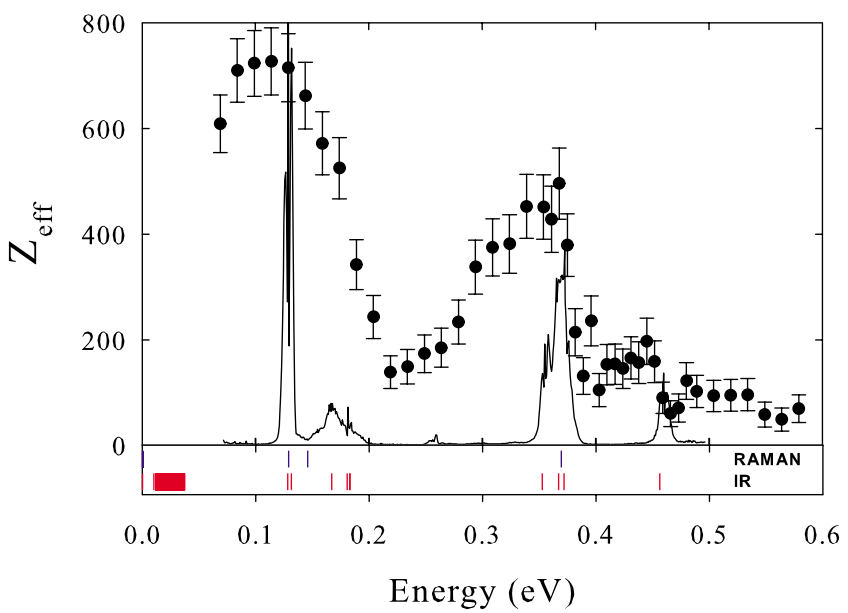

FIG. 2. (Color online) $Z_{\text {eff }}$ spectrum (solid circles) for methanol. Also shown is the normalized gas-phase IR absorption data (solid curve) and the locations of the IR- and Raman-active vibrational modes (vertical bars) from Ref. [16].

were measured, and the results are shown by open circles in Fig. 1. Now the theoretical predictions for the deuterated methyl halides have no fitted parameters, and so Fig. 1 represents an absolute comparison between theory and experiment. The agreement is very good in $\mathrm{CD}_{3} \mathrm{Cl}$, as is the agreement for the resonance magnitudes in $\mathrm{CD}_{3} \mathrm{Br}$. Structure at energies below $0.15 \mathrm{eV}$ in the theory curves is not fully resolved in the data. This may be due to vibrationally inelastic scattering on $\mathrm{C}$-halogen modes, which can alter the energy distribution of the positron beam for low energies and just above the inelastic thresholds, but this bears further scrutiny. The small energy shift between theory and experiment of $\sim 12 \mathrm{meV}$ for $\mathrm{CD}_{3} \mathrm{Br}$ is of the order of the estimated positron energy calibration, $\pm 10 \mathrm{meV}$.

The calculations shown in Fig. 1 include only the resonances due to fundamental vibrations, all of which are IR active. The agreement between the theory and experiment means that contributions from VFRs involving overtones or combination vibrations are either absent or very weak in these molecules.

However, we have now identified a number of small polyatomics in which combination or overtone resonances make large contributions to the $Z_{\text {eff }}$, in spite of weaker dipole couplings. Shown in Fig. 2 are $Z_{\text {eff }}$ and gas-phase IR absorption data for methanol $\left(\mathrm{CH}_{3} \mathrm{OH}\right)$. The smaller energy spacing between 350 and $450 \mathrm{meV}$ is due to the fact that the measurement was repeated to better resolve the high-energy features. Any resulting discontinuity is within the error bars. As compared with the methyl halides, the $\mathrm{C}-\mathrm{H}$ stretch peak at $\sim 360 \mathrm{meV}$ has more spectral weight on the low-energy side in a region with little IR activity. There is also a peak at $430 \mathrm{meV}$ that we associate with the $\mathrm{O}-\mathrm{H}$ stretch mode, which occurs at $456 \mathrm{meV}$ in the gas phase [16]. This difference may be due to the presence of the positron, since a similar O-H energy shift is observed in liquid phase. This could be evidence that positron binding changes the molecular vibrational frequency, an effect that is neglected by the present theory [7] 


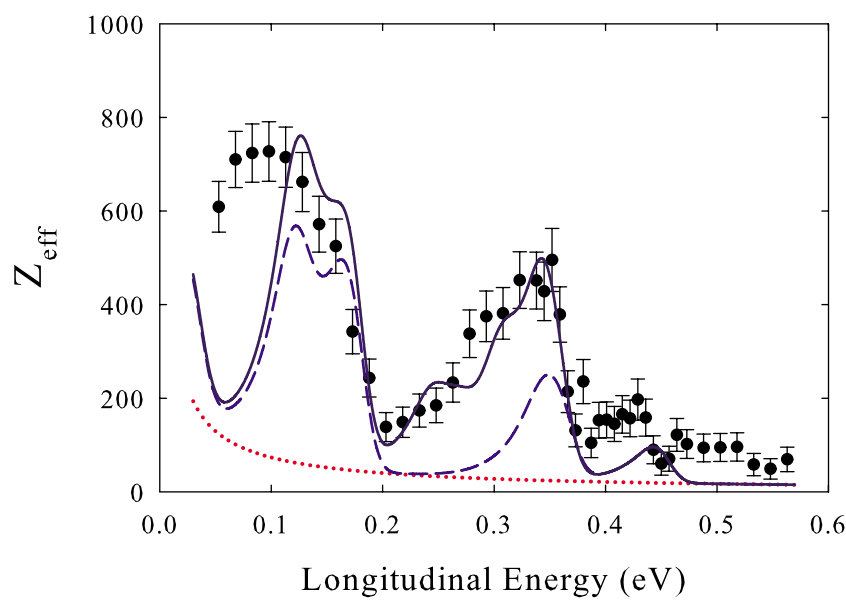

FIG. 3. (Color online) Comparison of measured $Z_{\text {eff }}$ for methanol (solid circles) with the theoretical predictions obtained using a fitted $\varepsilon_{b}=2 \mathrm{meV}$. The dotted curve is the nonresonant (direct) contribution (see Ref. [7]), the dashed curve includes only VFRs based on fundamentals, and the solid curve includes both the fundamental and multimode VFRs. See text for details.

All vibrational modes in methanol are IR active, making it an ideal application for the theory of Ref. [7]. In Fig. 3 the measured $Z_{\text {eff }}$ and theoretical predictions, using a fitted $\varepsilon_{b}$ $=2 \mathrm{meV}$, are compared. The vibrational energies, transition dipole moments, and capture widths for methanol are given in Table I. The dashed curve in Fig. 3 is the $Z_{\text {eff }}$ obtained using only VFRs based on the single modes. This results in a narrow $\mathrm{C}-\mathrm{H}$ stretch peak half the size of that measured and no VFR activity between this peak and $200 \mathrm{meV}$. Increasing $\varepsilon_{b}$ does not resolve this discrepancy, because it makes the low-energy peak too large, and still cannot reproduce the shape of the $0.2-0.35 \mathrm{eV}$ peak.

This discrepancy can be resolved if one considers weaker resonances due to overtones and combinations detected in the IR absorption spectra near $0.25 \mathrm{eV}$ [17] (see Fig. 2 and Table I). The elastic widths of these resonances are in general smaller than those of the modes. However, since $\Gamma_{\nu}^{e} \gg \Gamma_{\nu}^{a}$, their contributions to $Z_{\text {eff }}$ are similar. Including these multimode resonances results in an enhanced magnitude and width of $Z_{\text {eff }}$ around the $\mathrm{C}-\mathrm{H}$ stretch peak (solid curve in Fig. 3). In fact, their integral contribution in the $0.2-0.35 \mathrm{eV}$ range is approximately twice that of the modes, with a smaller addition to the low energy peaks. Theory and experiment are now in much closer agreement above $0.1 \mathrm{eV}$. The major remaining discrepancy is the relatively small theoretical $Z_{\text {eff }}$ at lower energies. The calculation includes a torsion mode at $\sim 40 \mathrm{meV}$, but its contribution is insufficient to fill in the gap up to the next higher-energy peak. The C-O-H torsion vibrations are strongly coupled to rotations [16]. This could lead to additional low-energy multimode VFRs, but we have no estimate of this effect.

This analysis for methanol provides direct, quantitative evidence that multimode VFRs can make significant contributions to the $Z_{\text {eff }}$ spectra, particularly in the $0.2-0.3 \mathrm{eV}$ energy range. With this in mind, we reexamined the $Z_{\text {eff }}$ spectra of other small hydrocarbons, finding that acetylene, ethylene, ethane, and cyclopropane [3-5] all exhibit contri-
TABLE I. Parameters of positron VFRs in methanol.

\begin{tabular}{|c|c|c|c|c|}
\hline$\nu$ & Vibrations $^{\mathrm{a}}$ & $\begin{array}{c}\omega_{\nu}^{\mathrm{b}} \\
(\mathrm{meV})\end{array}$ & $\begin{array}{c}d_{\nu}^{\mathrm{c}} \\
\text { (a.u.) }\end{array}$ & $\begin{array}{c}\Gamma_{\nu}^{e \mathrm{~d}} \\
(\mu \mathrm{eV})\end{array}$ \\
\hline 1 & $\nu_{1}$ & 456 & 0.026 & 59 \\
\hline 2 & $\nu_{2}$ & 369 & 0.024 & 43 \\
\hline 3 & $\nu_{3}$ & 351 & 0.023 & 38 \\
\hline 4 & $\nu_{4}$ & 183 & 0.016 & 12 \\
\hline 5 & $\nu_{5}$ & 180 & 0.011 & 5.8 \\
\hline 6 & $\nu_{6}$ & 176 & 0.040 & 75 \\
\hline 7 & $\nu_{7}$ & 138 & 0.026 & 27 \\
\hline 8 & $\nu_{8}$ & 128 & 0.074 & 209 \\
\hline 9 & $\nu_{9}$ & 365 & 0.029 & 62 \\
\hline 10 & $\nu_{10}$ & 183 & 0.016 & 12 \\
\hline 11 & $\nu_{11}$ & 135 & 0.009 & 3.0 \\
\hline 12 & $\nu_{12}$ & 36.6 & 0.087 & 115 \\
\hline 13 & $2 \nu_{5}, \nu_{4}+\nu_{6}$ & 360 & 0.027 & 53 \\
\hline 14 & $\nu_{5}+\nu_{6}$ & 357 & 0.012 & 10 \\
\hline 15 & $2 \nu_{6}$ & 348 & 0.024 & 41 \\
\hline 16 & $\nu_{7}+\nu_{10}$ & 322 & 0.009 & 5.0 \\
\hline 17 & $\nu_{8}+\nu_{10}$ & 313 & 0.016 & 17 \\
\hline 18 & $2 \nu_{7}$ & 276 & 0.006 & 2.2 \\
\hline 19 & $2 \nu_{8}$ & 254 & 0.008 & 3.8 \\
\hline 20 & - & 161 & 0.017 & 13 \\
\hline 21 & - & 149 & 0.018 & 14 \\
\hline
\end{tabular}

${ }^{a}$ Identification of vibrations from Ref. [17]. For all states $b_{\nu}=1$.

${ }^{\mathrm{b}}$ Energies of fundamentals (1-12) and combinations and/or overtones (13-21) from Ref. [17], except $\nu_{1}$ and $\nu_{12}$ [16].

${ }^{\mathrm{c}}$ Values from Ref. [17], except $\nu_{1}$ [18].

${ }^{\mathrm{d}}$ Values obtained for $\varepsilon_{b}=2 \mathrm{meV}$, which yields $\Gamma_{\nu}^{a}=0.042 \mu \mathrm{eV}$.

butions from multimode VFRs. In contrast to the substituted methanes, only some of the modes in these molecules are IR active. Thus there is no simple way to calculate the capture widths for all VFRs including overtones or combinations. Nevertheless, their effect can be estimated by using Eq. (2) and assuming that $\Gamma_{\nu}^{e} \gg \Gamma_{\nu}^{a}$.

Shown in Fig. 4 are the results for ethylene. A binding energy of $10 \mathrm{meV}$ has been assumed, based on the position of the experimental $\mathrm{C}-\mathrm{H}$ stretch peak. Apart from the five IR-active modes (i.e., those with $B_{u}$ symmetry), and six other modes $\left(A_{g}\right.$ and $\left.B_{g}\right)$ that can be populated by $s, p$, and $d$ wave positrons, the contributions of 14 known IR-active combination vibrations identified in Ref. [19] are included. For the latter VFRs we set $\Gamma_{\nu}^{e} / \Gamma_{\nu}=1 / n$ empirically, where $n$ is the number of single vibrational quanta in the combination, to best match the observed $Z_{\text {eff }}$ spectrum [20]. Figure 4 shows that $Z_{\text {eff }}$ due to the $B_{u}$ modes considerably underestimates the experimental results. Adding VFRs of $A_{g}$ and $B_{g}$ modes improves the agreement near the peaks, but the annihilation signal in the $0.2-0.3 \mathrm{eV}$ range is still poorly described. As shown in Fig. 4, the theory can match the experiment only by allowing for combination VFRs, which contribute most of the spectral weight above $0.2 \mathrm{eV}$. 


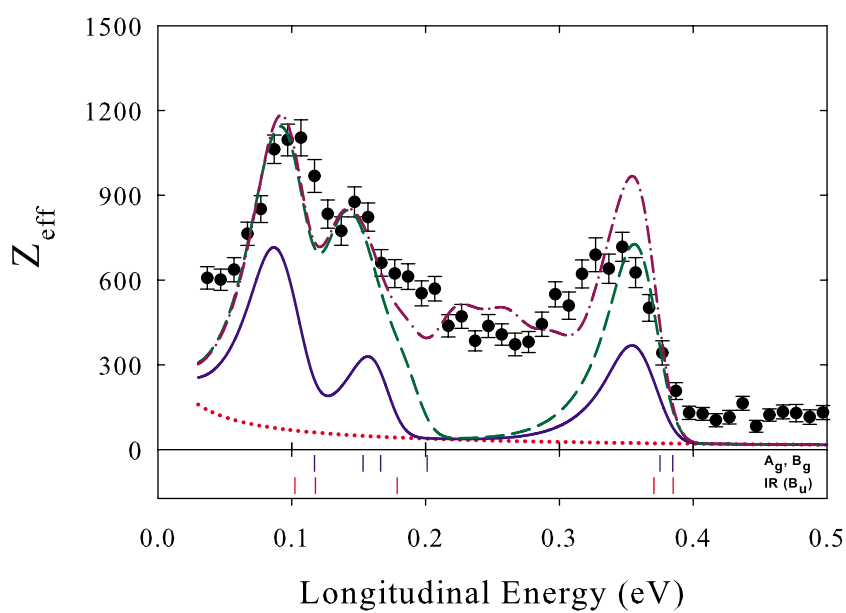

FIG. 4. (Color online) Comparison of $Z_{\text {eff }}$ measured for ethylene (solid circles) with $Z_{\text {eff }}$ calculated for $\varepsilon_{b}=10 \mathrm{meV}$ using IR $\left(B_{u}\right)$ modes only (solid); $A_{g}, B_{u}$, and $B_{g}$ modes (dashed line); these plus IR-active combinations (dot-dashed line), in addition to direct annihilation (dotted line). Vertical bars show the mode energies.

In larger molecules, $Z_{\text {eff }}$ increases rapidly with molecular size but distinct multimode resonances are generally not observed $[3-5,8]$. The increases in $Z_{\text {eff }}$ can be explained by intramolecular vibrational relaxation (IVR), which involves

[1] G. F. Gribakin, Phys. Rev. A 61, 022720 (2000).

[2] G. Gribakin, in New Directions in Antimatter Physics and Chemistry, edited by C. M. Surko and F. A. Gianturco (Kluwer Academic, Dordrecht, 2001), pp. 413-435.

[3] S. J. Gilbert, L. D. Barnes, J. P. Sullivan, and C. M. Surko, Phys. Rev. Lett. 88, 043201 (2002).

[4] L. D. Barnes, S. J. Gilbert, and C. M. Surko, Phys. Rev. A 67, 032706 (2003).

[5] L. D. Barnes, J. A. Young, and C. M. Surko, Phys. Rev. A 74, 012706 (2006).

[6] H. Hotop, M.-W. Ruf, M. Allan, and I. I. Fabrikant, Adv. At., Mol., Opt. Phys. 49, 85 (2003).

[7] G. F. Gribakin and C. M. R. Lee, Phys. Rev. Lett. 97, 193201 (2006).

[8] J. A. Young and C. M. Surko, Phys. Rev. Lett. 99, 133201 (2007).

[9] D. A. L. Paul and L. Saint-Pierre, Phys. Rev. Lett. 11, 493 (1963).

[10] G. R. Heyland, M. Charlton, T. C. Griffith, and G. L. Wright, Can. J. Phys. 60, 503 (1982).

[11] C. M. Surko, A. Passner, M. Leventhal, and F. J. Wysocki, coupling of mode-based doorway resonances to other vibrational excitations (including multimode excitations) $[3,4,15,8]$. It is presently unclear whether the multimode resonances observed in small molecules are absent in larger molecules or are simply obscured by more strongly enhanced single-mode resonances.

In summary, the theory provides good quantitative predictions for the magnitudes, positions, and shapes of dipoleallowed, single-mode VFR peaks in many small molecules, in some cases, with no fitted parameters. By applying it to molecules such as methanol and ethylene, we find strong evidence that multimode and other dipole-forbidden VFRs are important and that their effects can be quantified. However, obstacles remain in predicting the strengths of the VFRs of IR-inactive vibrations.

We note that there is also some evidence of multimode VFR in electron attachment to molecules [6]. With few exceptions (e.g., $\mathrm{N}_{2} \mathrm{O}[21]$ ), these features are relatively weak and difficult to identify. In contrast, in the positron case, the annihilation channel puts weakly coupled, multimode attachment on an equal footing with dipole-allowed single-mode effects [cf. Eq. (1)], thereby enhancing significantly the contributions of multimode effects.

This work is supported by National Science Foundation Grant No. PHY-02-44653.

Phys. Rev. Lett. 61, 1831 (1988).

[12] The formula for $\Delta(E)$, Eq. (10) of Ref. [7], must contain an extra factor $1 / 2$ on the right-hand side. The correct expression was used to obtain the results in Ref. [7].

[13] L. M. Bishop and L. M. Cheung, J. Phys. Chem. Ref. Data 11, 119 (1982).

[14] C. M. R. Lee, Ph.D. thesis, Queen's University, Belfast, 2006 (unpublished).

[15] G. F. Gribakin and P. M. W. Gill, Nucl. Instrum. Methods Phys. Res. B 221, 30 (2004).

[16] NIST Chemistry WebBook (2005), http://webbook.nist.gov/ chemistry/

[17] J. E. Bertie and S. L. Zhang, J. Mol. Struct. 333, 413 (1997).

[18] J. Florian, J. Leszczynski, B. G. Johnson, and L. Goodman, Mol. Phys. 91, 439 (1997).

[19] R. Georges, M. Bach, and M. Herman, Mol. Phys. 97, 279 (1999).

[20] A possible physical reason for this is that combination vibration VFRs can have more than one positron emission channel, increasing their total widths, so that $\Gamma_{\nu}^{e}<\Gamma_{\nu}$.

[21] M. Allan and T. Skalický, J. Phys. B 36, 3397 (2003). 